\title{
Vorwort
}

\section{zur zweiten Auflage}

Die vorliegende Beschreibung der Gigantomachie ist von O. Puchstein verfaßt und für die neue Auflage von ihm und $\mathrm{H}$. Winnefeld noch einmal durchgesehen. Sie ist ausgearbeitet mit Benutzung der seit 1880 mehrmals gedruckten "Beschreibung der pergamenischen Bildwerke «, deren erster Entwurf von A. Conze herrührt, und auf Grund der beiden, in den Sitzungsberichten der Königlichen Akademie der Wissenschaften 1888 und r 889 veröffentlichten Abhandlungen, durch welche Puchstein selbst die Anordnung der die Gigantomachic bildenden Relicfplatten und in allen wesentlichen Teilen die Deutung festgelegt hat. Nur an zwei Stellen haben die praktischen Versuche bei Aufstellung der Platten im neuen Pergamon-Museum zu Abweichungen von Puchsteins Anordnung geführt: das von ihm zu Hemera gerechnete obere Ende einer Platte ist neben Hera gestellt und die arg zerstörte Darstellung auf Hebe bezogen worden, und die Figuren, die Puchstein nebeneinander geordnet und als zweite und dritte Gorgone gedeutet hatte, mußten getrennt werden; daß die dazwischen geschobene Gruppe, die früher ihren Platz zwischen Nyx und Erinys hatte, gerade hierher gehöre, ist wahrscheinlich, aber nicht $z u$ beweisen. Überhaupt zwang die Notwendigkeit der Aufstellung dazu in allen zweifelhaften Fällen, wo eine endgültige Entscheidung nicht zu finden war, eine vorläufige nach Wahrscheinlichkeitsgründen zu treffen. Die Beschreibung schließt sich der Aufstellung an. Die kleinen Bruchstücke, deren Einfügung bisher noch nicht gelungen ist, sind dabei fürs erste unberiicksichtigt geblieben.

Die Angaben über die Iage des einst mit der Gigantomachie geschmückten Altars und über dessen architektonische Wiederherstellung sind meist den vorläufigen Berichten über die Ergebnisse der Ausgrabungen zu Pergamon (Jahrbuch der Königlich Preußischen Kunstsammlungen I880, I882, 1888) entnommen.

Die Vorlagen für die der Beschreibung beigefügten Abbildungen sind mit Hilfe photographischer Aufnahmen von M. Lübke unter Aufsicht von Puchstein und F. Winter hergestellt, die für die neue Auflage nötigen Änderungen und $\mathrm{Zu}$ sätze von M. Lübke nach Angabe von Winnefeld ausgeführt worden. Diese Abbildungen machen keinen Anspruch darauf, die stilistische Eigentümlichkeit und 
Vorwort.

die Wirkung des großen Denkmals, das sich in einem über das erste Hoffen weit hinausgehenden Maße in der Werkstatt der Königlichen Museen wiederherstellen ließ, wiederzugeben. Sie sollen nur die Kenntnis des Tatsächlichen vermitteln und anschaulich machen. Demselben Zwecke dienen die beiden Übersichtstafeln I und II. Auf Tafel III und IV sind die auf die Gigantomachie bezüglichen Inschriften aus Band VIII der "Altertümer von Pergamon " wiederholt und dabei so weit wie möglich so angeordnet worden, wie sie der Reihe nach sich auf dem Fries folgten.

\section{Der Direktor}

der Sammlung antiker Skulpturen bei den Königlichen Museen

Kekule von Stradonitz 